\title{
Temperature dependence of piezoresistance of composite Fermions with a valley degree of freedom
}

\author{
T. Gokmen, Medini Padmanabhan, and M. Shayegan \\ Department of Electrical Engineering, Princeton University, Princeton, NJ 08544
}

(Dated: April 24, 2022)

\begin{abstract}
We report transport measurements of composite Fermions at filling factor $\nu=3 / 2$ in AlAs quantum wells as a function of strain and temperature. In this system the composite Fermions possess a valley degree of freedom and show piezoresistance qualitatively very similar to electrons. The temperature dependence of the resistance $(R)$ of composite Fermions shows a metallic behavior $(d R / d T>0)$ for small values of valley polarization but turns insulating $(d R / d T<0)$ as they are driven to full valley polarization. The results highlight the importance of discrete degrees of freedom in the transport properties of composite Fermions and the similarity between composite Fermions and electrons.

PACS numbers:
\end{abstract}

Since the discovery of the fractional quantum Hall effect (FQHE) [1], a great deal of research has been devoted to understand the ground state of a two-dimensional electron system (2DES) at high magnetic fields. Although Laughlin's original wave function successfully explained the first observed FQHE state at filling factor $\nu=1 / 3[2]$, it is the composite Fermion theory [3-6] that has unified the origin of nearly all the fractional states. Composite Fermions (CFs) are formed by attachment of an even number of magnetic flux quanta to each electron. At exact half-fillings the attached flux cancels out the external magnetic field and the CFs feel zero effective magnetic field. They are therefore expected to have Fermi liquid properties and, in particular, form a Fermi sea [5, 6]; this has indeed been verified in numerous experiments 7 -9].

Although the flux attachment cancels the external magnetic field at half-fillings, any spatial inhomogeneity in the density of electrons (because of the random impurity potential) results in a random, non-zero effective magnetic field that is seen by CFs. Such a random field is expected to suppress the weak localization effect and give rise to a metallic ground state for CFs in a low-disorder 2DES [4]. With increasing disorder, the CF system can be driven through a metal-insulator transition (MIT) [4]. Indeed, for CFs at $\nu=1 / 2$ a metallic temperature dependence at high densities and a disorder-induced MIT (via lowering the density) was experimentally demonstrated [10].

Here we report piezoresistance measurements for CFs at $\nu=3 / 2$ in an AlAs quantum well 2DES. In this system, the CFs possess a valley degree of freedom [11], and their valley occupation can be controlled via the application of in-plane strain. Our piezoresistance traces at $\nu=3 / 2$ show an increase in the resistance of CFs and a clear "kink" as the CFs make a two-valley to single-valley transition. This is qualitatively similar to the piezoresistance of electrons at zero magnetic field. The temperature dependence of the piezoresistance reveals that, like their electron counterparts [12], increasing valley polar- ization changes the sign of the temperature dependence of resistance of CFs signaling the importance of the discrete degrees of freedom in the transport properties of CFs.

We performed experiments on a 2DES confined to a $15 \mathrm{~nm}$ thick layer of AlAs, and modulation-doped with Si. Our sample was grown by molecular beam epitaxy on a (001) GaAs substrate. The electrons in this sample occupy two in-plane valleys with elliptical Fermi contours as shown in Fig. 1(b) [13], each centered at an X point of the Brillouin zone, and with an anisotropic effective mass (longitudinal mass $m_{l}=1.05$ and transverse mass $m_{t}=0.205$, in units of free electron mass). We refer to these valleys by the orientation of their major axis, [100] and [010]. To vary the occupations of the two valleys we glue our samples to a piezoelectric actuator (piezo), and apply voltage bias to the piezo to stretch the sample in one direction and compress it in the perpendicular direction [11 15]. This results in a symmetry breaking strain $\epsilon=\epsilon_{[100]}-\epsilon_{[010]}$, where $\epsilon_{[100]}$ and $\epsilon_{[010]}$ are the strain values along the $[100]$ and [010] directions. For $\epsilon>0$ electrons are transferred from the [100] valley to the [010] valley and vice-versa for $\epsilon<0$; in either case the total density remains fixed with strain. The resulting valley splitting energy is given by $E_{V}=\epsilon E_{2}$ where $E_{2}$ is the deformation potential which in AlAs has a band value of $5.8 \mathrm{eV}$ [13]. We use a metal-foil strain gauge glued to the opposite face of the piezo to measure the applied strain [13]. The lithographically defined Hall-bar mesa is aligned along the [110] direction to pass current at $45^{\circ}$ with respect to the major axes of the valleys so that the antisymmetric piezoresistance due to mass anisotropy [15] is minimized. We used a top gate to vary the electron density $(n)$. All measurements were done in a dilution refrigerator with a base temperature $(T)$ of $20 \mathrm{mK}$ and an $18 \mathrm{~T}$ superconducting magnet.

In Fig. 1(a) we show resistance $(R)$ vs. magnetic field (B) data at $n=5.47 \times 10^{11} \mathrm{~cm}^{-2}$ for $\epsilon=0$ (balanced valleys). In addition to the integer quantum Hall states 
at low $B$, well-developed FQHE states around $\nu=3 / 2$ such as $\nu=5 / 3,4 / 3,8 / 5$ and $7 / 5$ (and also in the second Landau level at $\nu=8 / 3$ and $7 / 3$ ) can be seen. After determining the density and the position of $\nu=3 / 2$ from magnetoresistance data, we take piezoresistance traces at $B=0$ and at $\nu=3 / 2$ as shown in Figs. 1(d) and (e). Each trace in these figures is normalized to the value of resistance at $\epsilon=0$ and shifted by 0.3 units vertically for clarity. In the remainder of the paper we first discuss the piezoresistance data taken at $B=0$ and then come back to $\nu=3 / 2$. Finally, we present data on the temperature dependence of the piezoresistance.

The Fermi contour of the electrons at zero strain, and the $B=0$ piezoresistance data for a range of densities are shown in Figs. 1(b) and (d), respectively. The piezoresistance data show two noteworthy features: First, the resistance increases as strain is swept away from zero. Second, at high values of strain the resistance shows a kink following which it changes very slowly. (Traces taken at high densities do not show the kink because of our limited strain range.) The kink positions in the piezoresistance traces mark the onset of full valley polarization of electrons, as documented previously [12]. The increase in resistance is because of the transfer of electrons between the valleys and can be understood reasonably well by incorporating the anisotropic effective mass of electrons [16] and the role of screening and scattering. For the current configuration shown in Fig. 1(b) the prediction of a simple Drude model, which adds the conductivities of the two valleys with an effective mass anisotropy ratio of $r=m_{l} / m_{t}=5.12$ and assumes a fixed scattering time, is shown in Fig. 1(d) with a dashed line. This curve is only adjusted to match the kink position and the resistance minimum of the $n=3.93 \times 10^{11} \mathrm{~cm}^{-2}$ data. This model predicts the ratio of the resistances from saturation to balance to be $R_{e}^{[110]}=(r+1)^{2} / 4 r=1.83$. We point out that there might be additional contributions from screening and scattering effects. Screening becomes less effective with increasing valley polarization and can cause an extra increase in the resistance at larger valley polarizations [12, 17]. In contrast to screening, the intervalley scattering is more pronounced when two valleys are occupied and results in a larger resistance around balance. Since the experimentally measured values of $R_{e}^{[110]}$ range from 1.8 to 2.1 and the data show a faster rise in resistance with strain compared to the simple Drude model, we conclude that the piezoresistance at $B=0$ mainly comes from the mass anisotropy and the loss of screening.

The energy level diagram at $\nu=3 / 2$ for balanced valleys and piezoresistance of CFs are shown in Figs. 1(c) and (e), respectively. At high densities, the piezoresistance of CFs exhibits features qualitatively similar to the piezoresistance of electrons: the resistance increases as strain is swept away from zero and then saturates at
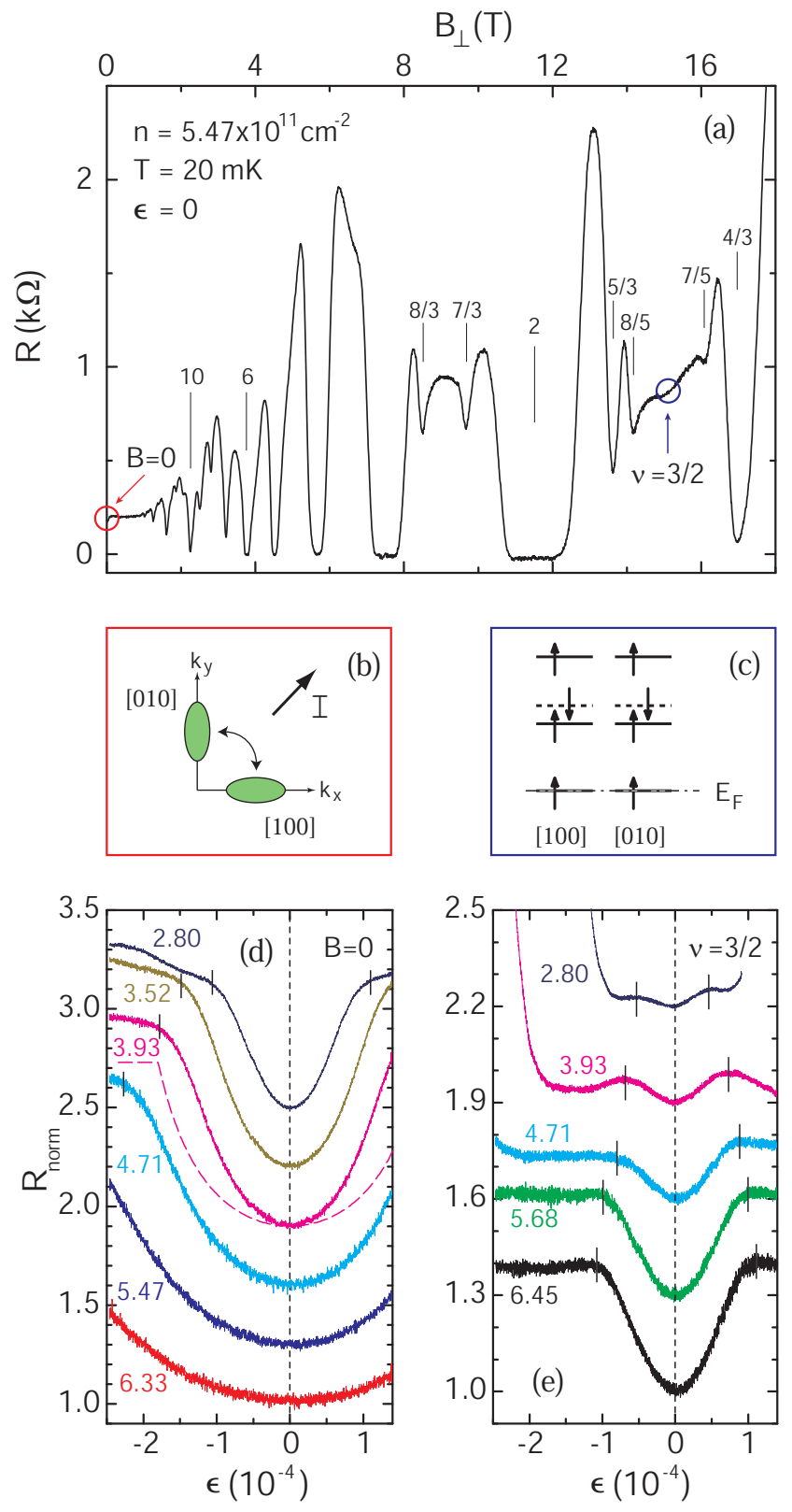

FIG. 1: (Color online) (a) Magnetoresistance ( $R$ vs. $\left.B_{\perp}\right)$ trace at zero strain (balanced valleys). (b) Schematic showing the Fermi contours of the electrons in two valleys and the strain induced inter-valley electron transfer. The current $(I)$ is applied along [110]. (c) Energy level diagram at $\nu=3 / 2$ for balanced valleys. (d) and (e) Piezoresistance traces at different densities for electrons and CFs, respectively. The values of density are given in units of $10^{11} \mathrm{~cm}^{-2}$, and the positions of the "kinks" are marked by vertical lines.

high strain values, signaling the full valley polarization of CFs. However, there are several differences. The resistance ratio $R_{C F}^{[110]}$ for $\mathrm{CF}$ piezoresistance from balance to saturation is smaller than $R_{e}^{[110]}$ and the kink occurs at smaller strain values for CFs compared to electrons. Furthermore, at low densities, we observe a dramatic rise in 
the piezoresistance at higher strains beyond the kink. We address these points in the following three paragraphs.

By making an analogy to electrons, the kink position in the $\nu=3 / 2$ piezoresistance traces can be associated with the full valley polarization of $\mathrm{CFs}$, and the strain value at the kink position gives the valley splitting energy that is equal to the Fermi energy of CFs. Since the CF Fermi sea is a direct manifestation of the Coulomb interaction, the valley splitting energy needed to valley polarize the CFs is determined by the Coulomb energy which is quantified by the magnetic length [11, 18]. The kink positions at $\nu=3 / 2$ piezoresistance traces in Fig. 1(e) are indeed in agreement with the results of previous studies of $\mathrm{CF}$ valley polarization energies, determined from coincidence measurements of CF Landau levels [11, 18].

The experimentally measured value of the resistance ratio $R_{C F}^{[110]}$ for CFs from balance to saturation is 1.4 for the highest density, and drops almost to unity for the lowest densities. We note that the experimentally measured value of $R_{C F}^{[110]}$ is smaller than $R_{e}^{[110]}$. Similar to $R_{e}^{[110]}$, we expect that $R_{C F}^{[110]}$ would be affected by the effective mass anisotropy of CFs and the screening/scattering effects. Our piezoresistance resistance measurements along the [100] direction indeed show that CFs inherit the transport anisotropy of electrons at zero field, suggesting an anisotropy in CF effective mass [19]. However, we emphasize that the observed transport anisotropy of CFs along the [100] is smaller compared to electrons and therefore qualitatively in agreement with the observation of $R_{C F}^{[110]}<R_{e}^{[110]}$.

Another feature of the piezoresistance traces at $\nu=$ $3 / 2$ for low densities is the significant increase in resistance for high strains. The reason for this increase is the coincidence of the electron Landau levels. As can be surmised from Fig. 1(c), for sufficiently large strains, when the valley splitting energy is equal to the electron cyclotron energy, the lowest electron Landau level of one valley coincides with the second Landau level of the other valley. Beyond this coincidence, the electrons are fully valley polarized. Note that the increase in resistance at $\nu=3 / 2$ occurs roughly at the same strain value where the $B=0$ piezoresistance traces show the kink and signal full electron valley polarization. We add that at much higher strains, well past the coincidence, the piezoresistance at $\nu=3 / 2$ saturates again, consistent with the $B=0$ data.

Now we present the temperature dependence of the CF piezoresistance at $\nu=3 / 2$. In Figs. 2(b) and 2(c) we show piezoresistance traces for CFs at two densities. The data in Fig. 2(b) reveal that, at high densities and for small strains so that the valley polarization is small, CFs exhibit a metallic behavior $(d R / d T>0)$. With increasing strain, however, the resistance turns insulating $(d R / d T<0)$ as CFs become valley polarized. This observation demonstrates the importance of the discrete degrees of freedom for the temperature dependence of resistance of CFs. At lower densities (Fig. 2(c)) the metallic behavior around zero strain disappears, and the CFs act insulating in the full strain range, including at $\epsilon=0$ where they are valley degenerate.

Before presenting more details of our data, we briefly discuss the temperature dependence of the resistance of 2D electrons and the related MIT. The scaling theory predicts an insulating phase for a non-interacting 2DES with arbitrarily weak disorder, thanks to the weak localization of electrons [20]. However, experiments in high quality Si 2DESs [21] showed that, at high densities, the 2DES exhibits a metallic temperature dependence, and that the system can be driven to an insulating phase by lowering the 2DES density (increasing the disorder). Similar behavior has been reported for several other 2DESs 22]. Although there is no consensus whether or not there exists a true metallic ground state (in the limit of zero temperature) for a low-disorder 2DES, it is generally believed that the observed metallic behavior is due to the interplay of disorder, interaction and finite temperature effects. Additional measurements have also shown that the spin polarization of the 2DES plays a critical role in the apparent MIT: The system's behavior changes from metallic to insulating with increasing spin polarization [17]. A recent study [12] revealed that, for AlAs 2DESs, not only spin but also valley polarization is an important parameter for the apparent MIT, namely, the 2DES exhibits an insulating behavior when both valley and spin polarizations pass beyond some threshold value.

We illustrate this behavior for our AlAs 2DES sample in Fig. 2(a) where we show piezoresistance traces for electrons at a very high parallel magnetic field such that the $2 \mathrm{D}$ electrons are fully spin polarized $\left(B_{\perp}=0\right.$ in Fig. 2(a) trace). Both qualitatively and quantitatively our results are consistent with the results of Ref. [12]. At zero and small parallel fields, where the 2DES is not spin-polarized, it shows a metallic behavior in the entire strain range (data not shown). But when the 2DES is fully spin polarized via the application of a large parallel magnetic field, increasing the valley polarization changes the temperature dependence of resistance from metallic to insulating (Fig. 2(a)).

Our $\nu=3 / 2$ data in Fig. 2(b) show that the CFs qualitatively behave like the electrons. Note that because of the large g-factor, the Zeeman energy in our sample is comparable to and even larger than the cyclotron energy, as illustrated by the energy level diagram in Fig. $1(\mathrm{c})$. Therefore, the CFs at $\nu=3 / 2$ are fully spin polarized while we control their valley occupation via the application of strain. The electron data of Fig. 2(a) and CF data of Fig. 2(b) imply that, when the spin degree of freedom is frozen, (high density) electrons and CFs both show a metallic behavior when they have a valley degree of freedom. However, both systems show insulating behavior when the valley polarization is above some 

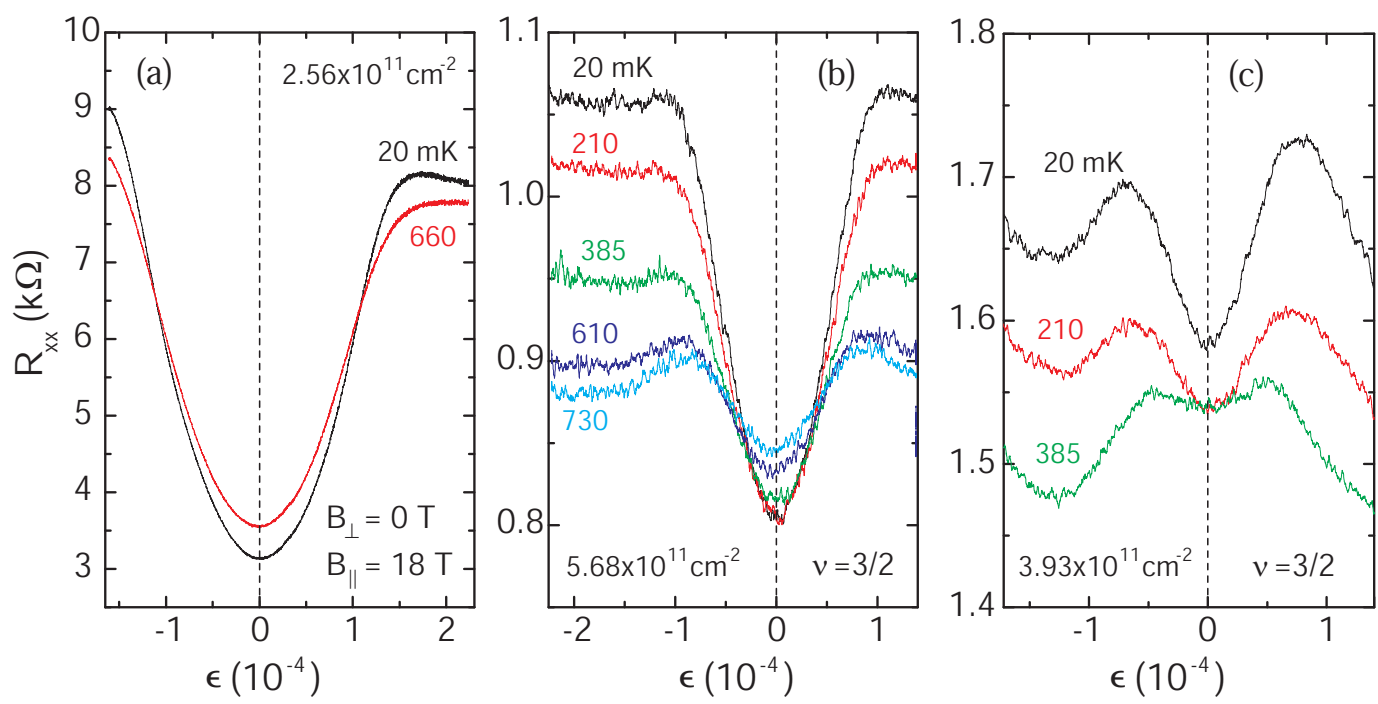

FIG. 2: (Color online) (a) Piezoresistance traces at different temperatures for spin polarized electrons. (b) and (c) Piezoresistance traces at different temperatures for CFs at $\nu=3 / 2$ for two different densities.

threshold value.

We observe the metallic behavior in the absence of strain and the valley polarization driven MIT for CFs in the high density range $\left(n>3.93 \times 10^{11} \mathrm{~cm}^{-2}\right)$. As the density is lowered, the metallic temperature dependence disappears and CFs exhibit an insulating behavior regardless of their valley polarization (Fig. 2(c)). This is consistent with the increasing disorder in the system at lower densities, and similar to the disorder-induced MIT of CFs demonstrated by Ref. [10]. We emphasize, however, that the CFs seem to be more sensitive to disorder than electrons: In the absence of strain, the density below which the electrons exhibit an insulating behavior is $\sim 1 \times 10^{11} \mathrm{~cm}^{-2}$ while for the $\nu=3 / 2 \mathrm{CFs}$ it is $\sim 4 \times 10^{11}$ $\mathrm{cm}^{-2}$.

Our piezoresistance results demonstrate that CFs and electrons show qualitatively very similar behaviors. However, they differ quantitatively in several ways. First, the valley splitting energy required to completely valley polarize the CFs is smaller than the electrons and this difference is reasonably well understood [11, 18]. Second, the piezoresistance ratio $R_{C F}^{[110]}$ from balance to saturation for CFs is smaller than the corresponding ratio $R_{e}^{[110]}$ for electrons and has a stronger temperature dependence. Third, the temperature dependence of the resistance, and the observation of an insulating behavior for CFs with increasing valley polarization or decreasing density, are qualitatively similar to electrons.

We thank the NSF for support. Part of this work was performed at the NHMFL, Tallahassee, which is also supported by the NSF. We thank E. Palm, T. Murphy, J. Park, and G. Jones for assistance.
[1] D.C. Tsui, H.L. Stormer, and A.C. Gossard Phys. Rev. Lett. 48, 1559 (1982).

[2] R.B. Laughlin Phys. Rev. Lett. 50, 1395 (1983).

[3] J.K. Jain, Phys. Rev. Lett. 63, 199 (1989).

[4] V. Kalmeyer and S.C. Zhang, Phys. Rev. B 46, 9889 (1992).

[5] B.I. Halperin, P.A. Lee, and N. Read, Phys. Rev. B 47, 7312 (1993).

[6] Composite Fermions, Jainendra K. Jain, Cambridge University Press, 2007.

[7] R.L. Willett, R.R. Ruel, K.W. West, and L.N. Pfeiffer, Phys. Rev. Lett. 71, 3846 (1993).

[8] W. Kang, H.L. Stormer, L.N. Pfeiffer, K.W. Baldwin, and K.W. West, Phys. Rev. Lett. 71, 3850 (1993).

[9] V.J. Goldman, B. Su, and J.K. Jain, , Phys. Rev. Lett. 72, 2065 (1994).

[10] C.T. Liang, J.E.F. Frost, M.Y. Simmons, D.A. Ritchie, and M. Pepper, Sol. Stat. Comm. 102, 327 (1997).

[11] N.C. Bishop, M. Padmanabhan, K. Vakili, Y.P. Shkolnikov, E.P. De Poortere, and M. Shayegan, Phys. Rev. Lett. 98, 266404 (2007).

[12] O. Gunawan, T. Gokmen, K. Vakili, M. Padmanabhan, E.P. De Poortere, and M. Shayegan, Nature Physics 3, 388 (2007).

[13] M. Shayegan, E.P. De Poortere, O. Gunawan, Y.P. Shkolnikov, E. Tutuc, and K. Vakili, Phys. Stat. Sol. (b) 243, 3629 (2006).

[14] O. Gunawan, Y.P. Shkolnikov, K. Vakili, T. Gokmen, E.P. De Poortere, and M. Shayegan, Phys. Rev. Lett. 97, 186404 (2006).

[15] Y.P. Shkolnikov, K. Vakili, E.P. De Poortere, and M. Shayegan, Appl. Phys. Lett. 85, 3766 (2004).

[16] G. Dorda, I. Eisele, and H. Gesch, Phys. Rev. B 17, 1785 (1978).

[17] This is analogous to the case of spin in many 2DESs where the resistance increases by a factor of up to 5 when 
the spin polarization changes from zero to one and screening is diminished [S. Das Sarma and E.H. Hwang, Phys. Rev. B 72, 205303 (2005)]. For experimental work, see: [D. Simonian et al., Phys. Rev. Lett. 79, 2304 (1997); T. Okamoto et al., Phys. Rev. Lett. 82, 3875 (1999); E. Tutuc et al., Phys. Rev. Lett. 86, 2858 (2001)].

[18] M. Padmanabhan, T. Gokmen, and M. Shayegan, Phys. Rev. B 80, 035423 (2009).

[19] T. Gokmen, M. Padmanabhan, and M. Shayegan, Nature
Physics 6, 621 (2010).

[20] E. Abrahams, P.W. Anderson, D.C. Licciardello, and T.V. Ramakrishnan, Phys. Rev. Lett. 42, 673 (1979)

[21] S.V. Kravchenko, G.V. Kravchenko, J.E. Furneaux, V.M. Pudalov, and M. DIorio, Phys. Rev. B 50, 8039 (1994).

[22] E. Abrahams, S.V. Kravchenko, and M.P. Sarachik, Rev. Mod. Phys. 73, 251 (2001). 